\title{
Transthoracic 3D Echocardiographic Imaging of Type A Aortic Dissection - Case Presentation
}

\author{
István Adorján Szabó, Ildikó Kocsis, Zoltán Fogarasi, Boglárka Belényi, Attila Frigy
}

Clinical County Hospital Mures, Romania

In type A aortic dissection (AoD) an early and accurate diagnosis is essential to improve survival, by applying urgent surgical repair. 3D transthoracic echocardiography (3D-TTE), an advanced noninvasive imaging technique, could offer a comprehensive evaluation of the ascending aorta and aortic arch in this regard. Both modalities of real-time 3D imaging - live 3D and full-volume aquisition - proved to be useful in evaluating the localization and extent of AoD. Our case illustrates the utility of 3D-TTE in the complex assessment AoD. By providing the proper anatomical dataset, 3D-TTE could facilitate considerably the diagnosis of type A AoD.

Keywords: type A aortic dissection, 3D transthoracic echocardiography

Received 7 June 2017 / Accepted 20 July 2017

\section{Introduction}

Recently, one of the most substantial developments in the field of echocardiography was the introduction of threedimensional (3D) imaging.

The advantages and clinical applications of this technique are multiple: (1) significant improvement in the quantitative evaluation of the cardiac chambers, including volumes and left ventricular ejection fraction, (2) better visualization of cardiac structures, like valves or congenital anomalies, (3) real-time control and guiding of percutaneous cardiac interventions (e.g., atrial septal defect closure), (4) evaluation of heart disease in the preoperative, intraoperative and postoperative setting, allowing immediate feedback on the effectiveness of surgical interventions. [1]

In our days, 3D echocardiography - due to the development of both hardware and software engineering - became a feasible, real-time examination method in the daily practice, using both the transthoracic and transesophageal approach.

The two main methods of examination in 3D echocardiography are live 3D mode and full volume acquisition with offline postprocessing.

During the live $3 \mathrm{D}$ examination a narrow pyramidal volume is exposed in real-time. This type of imaging has multiple advantages: (1) lack of stitching artefacts, (2) "true" real-time examination (of great value during interventions), (3) good temporal resolution, and (4) visualisation of different cardiac structures by steering the volume electronically. $[1,2]$

Our case represents an excellent example of the utility of transthoracic 3D echocardiography in the evaluation of a critical cardiac condition, a type A aortic dissection.

\section{Case presentation}

$\mathrm{KI}$, a 61 year old male, was referred to our department from a territorial hospital with the diagnosis of heart failure due to significant aortic regurgitation. At the admission, the patient complained of dyspnea with orthopnea, severe fatigue and a non-exertitional, non-anginal chest pain/discomfort. After a detailed history taking, we found that the first acute and severe chest pain appeared three months before, while brushing his teeth, and his clinical condition progressively worsened determining admissions to the territorial hospital. Physical examination revealed a severely distressed patient with pale and perspirated teguments, bilateral, basal crackles, and a 4/VI proto-mesodiastolic murmur at the Erb point accompanied by an ejection murmur, 5/VI, in the aortic area.

Routine 2D transthoracic echocardiographic examination (Fig. 1) revealed a severely dilated ascending aorta $(72 \mathrm{~mm})$, a severe aortic regurgitation and a great suspicion of aortic dissection, due to the existence of a linear structure in the aortic lumen, resembling with a dissection membrane.

The examination was continued with transthoracic 3D imaging, which confirmed the diagnosis of type A aortic dissection (Fig. 2, 3, 4) causing severe aortic regurgitation. The visualisation of the lesion was possible toward the thoracic descending aorta using the parasternal and suprasternal windows.

The patient was referred to the cardiovascular surgery clinic, but declined intervention, at hospital discharge being hemodynamically stable without signs of decompensation.

After 4 weeks he returned to our clinic with signs of severe cardiac decompensation. After stabilization he undervent cardiac surgery, but has died due to postoperative complications. 


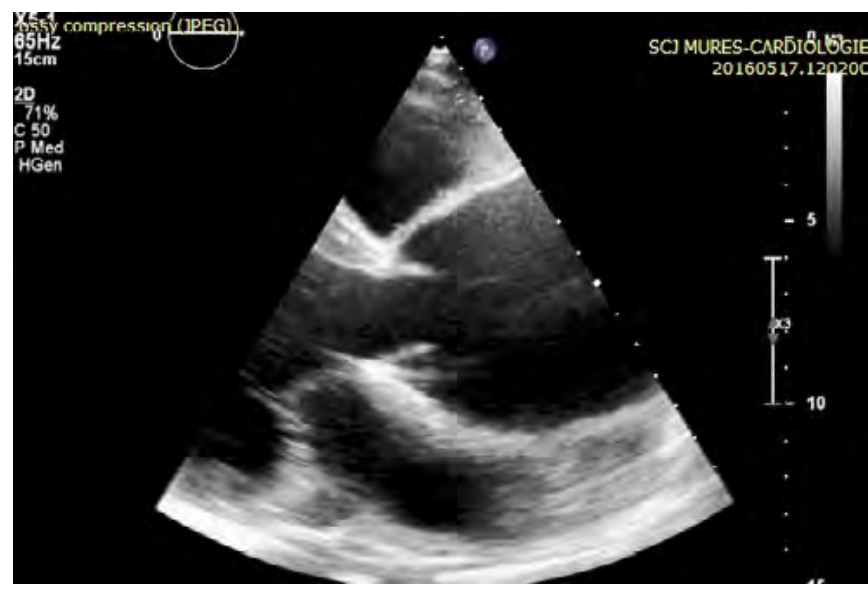

Fig. 1. 2D transthoracic echocardiography - parasternal long axis view of the ascending aorta: severe dilatation

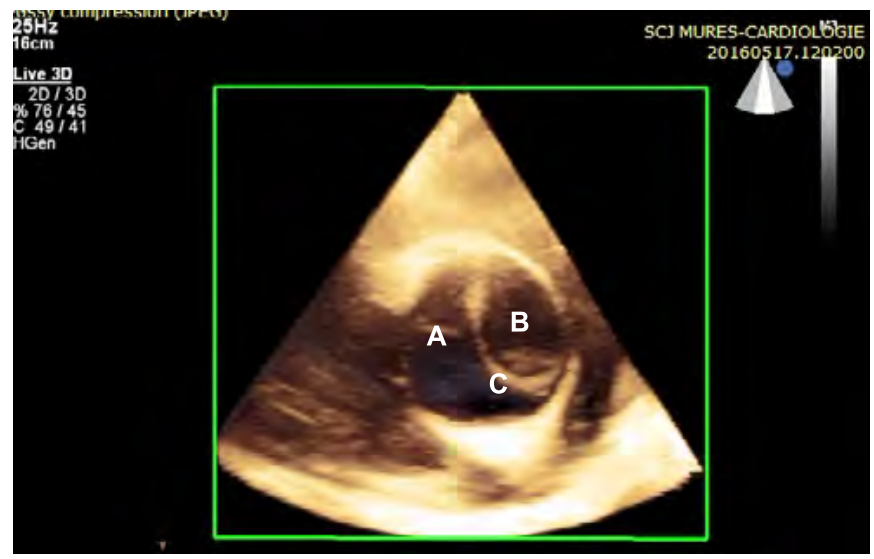

Fig. 3. 3D transthoracic echocardiography (live 3D) - parasternal short axis view (angulated superiorly): visualisation of the false (A) and true (B) lumen and the dissection membrane (C)

\section{Discussion}

Aortic dissection (AoD) is life threatening condition which occurs when blood enters into the media due to an intimal tear. Its incidence is estimated at 6:100.000 person per year, being higher in men than in women and increasing with age. The main risk factor associated with AoD is hypertension, which is present in almost $70 \%$ of patients. [3]

Timely diagnosis is important since the natural history of $\mathrm{AoD}$, if left untreated, includes a mortality as high as $2 \%$ per hour during the first two days, thus, urgent surgical repair repair could be lifesaver.

The ascending aorta (aortic root) is routinely evaluated during every standard echocardiographic examination, measuring the annulus and the sinusal, sino-tubular and tubular diameters. $[4,5]$

More precise diagnosis of AoD is based on computer tomography (angio CT) and two-dimensional transesophageal echocardiography (2D-TEE). CT is a feasible method (availability, instantaneous results), but cannot be performed at the bedside, also needing administration of a contrast agent. Echocardiography is the preferred method of examination in the unstable patient who is not transportable. Despite its incontestable usefulness, two-dimensio-

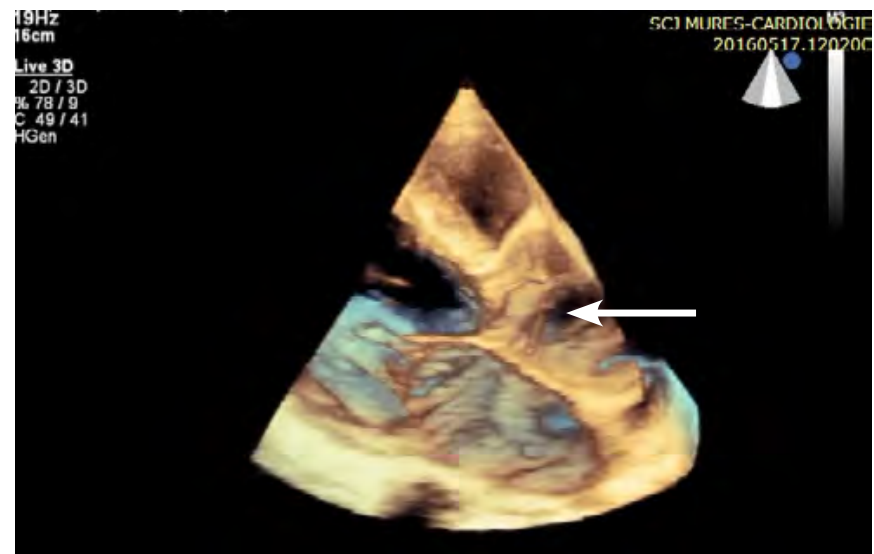

Fig. 2. 3D transthoracic echocardiography (live 3D) - parasternal long axis view: visualisation of the dissection membrane in the aortic lumen (arrow)

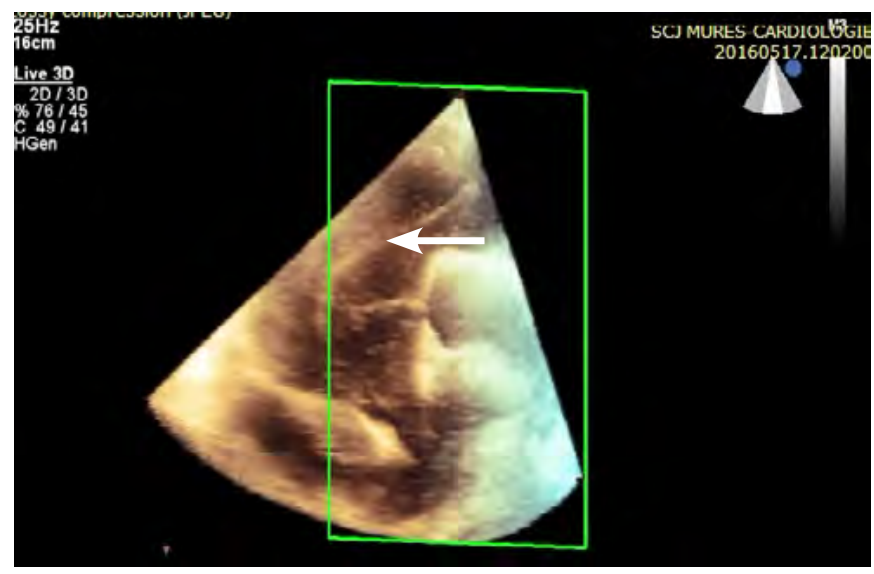

Fig.4. 3D transthoracic echocardiography (live 3D) - suprasternal long axis view of the aortic arch: visualisation of the dissection membrane (arrow)

nal transthoracic echocardiography (2D-TTE) has its own limitations regarding the visualisation of the whole aorta. Thus, in patients with adequate acoustic windows, realtime three-dimensional transthoracic echocardiography (3D-TTE) could provide all the relevant anatomical and functional data which make possible an accurate diagnosis of AoD, not requiring esophageal intubation or administration of intravascular contrast agent.

In our case, live 3D-TTE increased the accuracy of the diagnosis, since the dissection membrane was clearly seen on the 3D images. 3D-TTE has proven to be useful when AoD is suspected on 2DTTE, having an additional advantage, that makes possible the visualization of propagation into the branching vessels. [6-11]

Perspectively, in the diagnosis of aortic dissection, 3D-TTE is going to be more feasible, mainly because of the visualisation and measurements from the en face views, which provide more accurate and reproducible data in comparison with two-dimensional echocardiography. [1]

\section{Conclusions}

In our case, 3D transthoracic echocardiographic examination proved to be an accurate choice for diagnosing type A 
aortic dissection, conferring all the relevant anatomical and functional data needed for planning surgical repair.

\section{Ethical approval}

Approval of the Ethical Committee of Clinical County Hospital Mures (no. 3865/01.03.2016) was obtained for data processing and publication.

\section{Conflict of interest}

None to declare.

\section{References}

1. Lang MR, Badano LP, Tsang W, et al. EAE/ASE recommendations for image acquisition and display using three-dimensional echocardiography. J Am Soc Echocardiogr. 2012 Jan;25:3-46.

2. Vegas A. Three-dimensional transesophageal echocardiography: Principles and clinical applications. Ann Card Anaesth. 2016 Oct;19(Supplement):S35-S43.

3. Di Eusanio M, Trimarchi S, Patel $\mathrm{HJ}$, et al. Clinical presentation, management, and short-term outcome of patients with type $A$ acute dissection complicated by mesenteric malperfusion: observations from the International Registry of Acute Aortic Dissection. J Thorac Cardiovasc Surg. 2013 Feb;145:385-90.

4. Januzzi JL, Eagle KA, Cooper JV, et al. Acute aortic dissection presenting with congestive heart failure: results from the International Registry of Acute Aortic Dissection. J Am Coll Cardiol. 2005 Aug;46:733-5.

5. Flachskampf FA, Wouters PF, Edvardsen T, et al. Recommendations for transoesophageal echocardiography: EACVI update 2014. Eur Heart J Cardiovasc Imaging. 2014 Apr;15:535-65.

6. Golledge J, Eagle KA. Acute aortic dissection. Lancet. 2008 Jul;372:5566.

7. Pretre R, Von Segesser LK. Aortic dissection. Lancet. 1997 May;349:1461-4.

8. Wang CJ, Rodriguez Diaz CA, Trinh MA. Use of real-time threedimensional transesophageal echocardiography in type $A$ aortic dissections: advantages of 3D TEE illustrated in three cases. Ann Card Anaesth. 2015 Jan-Mar;18:83-6.

9. Nanda NC, Khatri GK, Samal AK, et al. Three dimensional echocardiographic assessment of aortic dissection. Echocardiography. 1998 Nov; 15:745-54.

10. Htay T, Nanda NC, Agrawal G, et al. Live three-dimensional transthoracic echocardiographic assessment of aortic dissection. Echocardiography. 2003 Aug;20:573-7.

11. Yelamanchili $P$, Nanda NC, Patel V, et al. Definitive diagnosis of descending thoracic aortic dissection by real time/live threedimensional transthoracic echocardiography. Echocardiography. 2006 Feb;23:158-61. 